\title{
Upgrade of a data acquisition system for SciBar Cosmic Ray Telescope (SciCRT) at Mt. Sierra Negra, Mexico
}

\author{
Yoshinori Sasai ${ }^{*},{ }^{a}$ Tetsuya Kawabata, ${ }^{a}$ Masahiro Ikeno, ${ }^{b}$ Tomohisa Uchida, ${ }^{b}$ Manobu \\ Tanaka, ${ }^{b}$ Yutaka Matsubara, ${ }^{a}$ Yoshitaka Itow, ${ }^{a}$ Takashi Sako, ${ }^{a}$ Diego Lopez, ${ }^{a}$ Rikiya \\ Hikimochi, ${ }^{a}$ Kazuoki Munakata, ${ }^{c}$ Chihiro Kato,${ }^{c}$ Masayoshi Kozai, ${ }^{c}$ Takaaki \\ Nakajima, ${ }^{c}$ Yoshiaki Nakamura, ${ }^{c}$ Shoichi Shibata, ${ }^{d}$ Akitoshi Oshima, ${ }^{d}$ Hisanori \\ Takamaru, ${ }^{d}$ Hiroshi Kojima, ${ }^{e}$ Harufumi Tsuchiya, ${ }^{f}$ Kyoko Watanabe,${ }^{g}$ Tatsumi Koi, ${ }^{,}$ \\ J.F.Valdés-Galicia, ${ }^{i}$ Ernesto Ortiz, ${ }^{i}$ Octavio Musalem, ${ }^{i}$ Alejandro Hurtado, ${ }^{i}$ Rocio \\ Garcia, ${ }^{i}$ Marcos Anzorena, ${ }^{i}$ Roberto Taylor, ${ }^{i}$ Marco Barrantes, ${ }^{i}$ and L.X.González, ${ }^{j}$ \\ E-mail: sasaiyoshinoriestelab.nagoya-u.ac.jp
}

SciCRT (SciBar Cosmic Ray Telescope) is a new project to observe cosmic rays via a full active scintillator tracker. Our aim is to detect high energy solar neutrons produced by the interaction between accelerated ions and the solar atmosphere and to observe muons to research anisotropy of Galactic Cosmic Rays (GCRs). In the previous ICRC in Brazil, we reported that the detector has been installed at Mt. Sierra Negra (4,600 m above sea level) in April 2013. We also reported that the current VME-based data acquisition (DAQ) system does not have enough capacity to deal with GCR-induced neutrons at such a high altitude mountain. Moreover noises produced by the VME readout prevent the DAQ system counting the trigger rate and make the current trigger process complicated.

Therefore we have developed a fast readout DAQ system, optimized to our experimental environment within an Open-It project. We employed SiTCP, a hardware-based network processor, developed for high energy physics. We have developed a brand-new back-end board (BEB) based SiTCP and tested it at Mt. Sierra Negra in 2014. Then we determined the definitive design for the new BEB. We replaced the muon and a part of the neutron DAQ system with the new DAQ system in June, 2015. We will introduce the basic performance evaluation and the configuration of the new DAQ system which is operated at Mt. Sierra Negra.

The 34th International Cosmic Ray Conference,

30 July - 6 August, 2015

The Hague, The Netherlands

\footnotetext{
* Speaker.

¿ Solar-Terrestrial Environment Laboratory, Nagoya University, Furo-cho, Chikusa-ku, Nagoya 464-8601, JAPAN

${ }^{b}$ High Energy Accelerator Research Organization, KEK, 1-1 Oho, Tsukuba, Ibaraki 305-0801, JAPAN
} 


\section{High Energy Solar Neutrons and Galactic Cosmic-ray Muons}

Solar neutrons have been observed over the course of three decades through various ways of measurements. Solar neutrons are produced by the interaction of accelerated ions with the solar atmosphere. Neutrons are not affected by the interplanetary magnetic field and are more preferable than ions themselves to study the acceleration mechanism of ions at the Sun's surface. On the ground level, solar neutron telescopes and neutron monitors have detected several solar neutron events which are accompanied with solar flares. These events were caused by high energy solar neutrons with more than $\sim 100 \mathrm{MeV}$. On the other hand, low energy neutrons $(<100 \mathrm{MeV})$ have been detected by satellite-borne detectors ([1], [2]). So far more than 10 solar neutron events have been detected on the ground by the network of solar neutron telescopes and neutron monitors ([3], [4]). To obtain the energy spectrum of neutrons, the time of flight method has been used in most cases. Therefore it is expected to get more solar neutron events and to install a new telescope we can determine the energy spectrum of neutrons with sufficient accuracy.

GCRs are affected by the magnetic field of the interplanetary space producing the directional anisotropy of the intensity observed at the Earth. To observe the anisotropy gives us the information on the GCR transport in the heliosphere and the space weather in the vicinity of the Earth. The Global Muon Detector Network (GMDN) consists of four multi-directional muon detectors in Japan, Australia, Brazil and Kuwait. It has a gap in the viewing direction around the North American Continent. Therefore it is desired to fill the region by using a muon detector.

\section{SciCRT Detector and Readout Electronics}

SciCRT, fully active scintillator tracker, is based on the SciBar detector developed for the K2K neutrino oscillation experiment in Japan. The detector consists of 15,000 plastic scintillator bars coated with a titanium based reflector. Each 116 scintillator bars are arranged in a same plane so that two planes are orthogonally attached and glued. This combination of two planes is called layer.

There are two detection parts of the SciCRT roughly. One part, the topmost and the bottommost layers, are applied for the Muon detector with four-fold trigger condition and charged veto counters to the other part for neutron detection. We divide the central part into eight components defined as Super Blocks (SBs). We call them SB1 to SB8 from the top to the bottom.

Scintillation photons from each scintillator bar are readout by a 64 channel Multi-Anode PhotoMultiplier Tube (MAPMT) through a wave-length shifting fiber (WLS fiber). A front-end board attached to the MAPMT has a VA/TA Application Specific Integrated Circuit (ASIC). "VA" is a

\footnotetext{
${ }^{c}$ Department of Physics, Shinshu University, Asahi, Matsumoto 390-8621, JAPAN

${ }^{d}$ College of Engineering, Chubu University, Kasugai 487-8501, JAPAN

${ }^{e}$ Faculty of Engineering, Aichi Institute of Technology, Toyota 470-0392, JAPAN

${ }^{f}$ Japan Atomic Energy Agency, 2-4 Shirakata Shirane, Tokai-mura, Naka-gun, Ibaraki 319-1195, JAPAN

${ }^{g}$ National Astronomical Observatory of Japan, 2-21-1 Osawa, Mitaka, Tokyo 181-8588, JAPAN

${ }^{h}$ SLAC National Accelerator Laboratory, Menlo Park, CA 94025-7015, USA

${ }^{i}$ Instituto de Geofísica, Universidad Nacional Autónoma de México, México, D.F., MEXICO

${ }^{j}$ SCiESMEX, Instituto de Geofísica, Unidad Michoacán, Universidad Nacional Autónoma de México, 58190, Morelia, Michoacán. MEXICO
} 


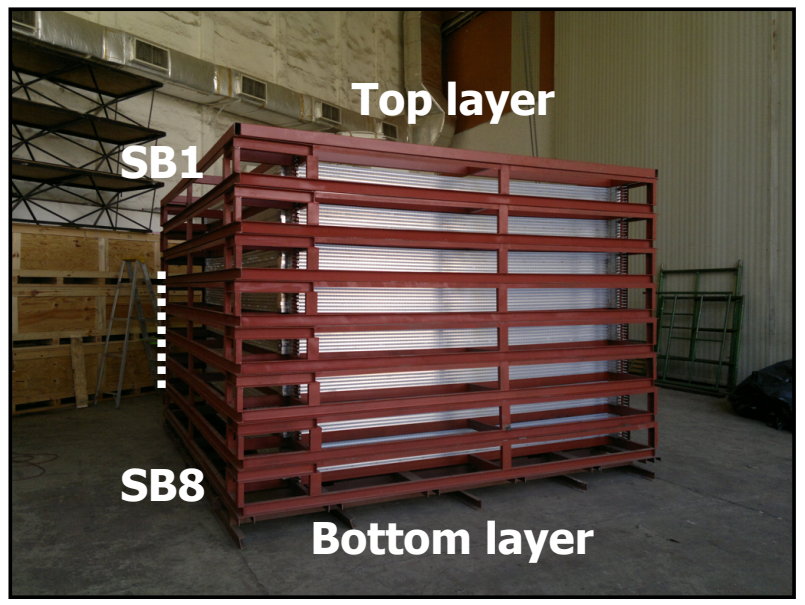

Figure 1: An image of the SciCRT detector. The detector is divided into two detection parts. One, the top and the bottom layers, are employed for the muon selection and the charged veto counter to the other part (SBs). Neutrons are accumulated using the central target SBs.

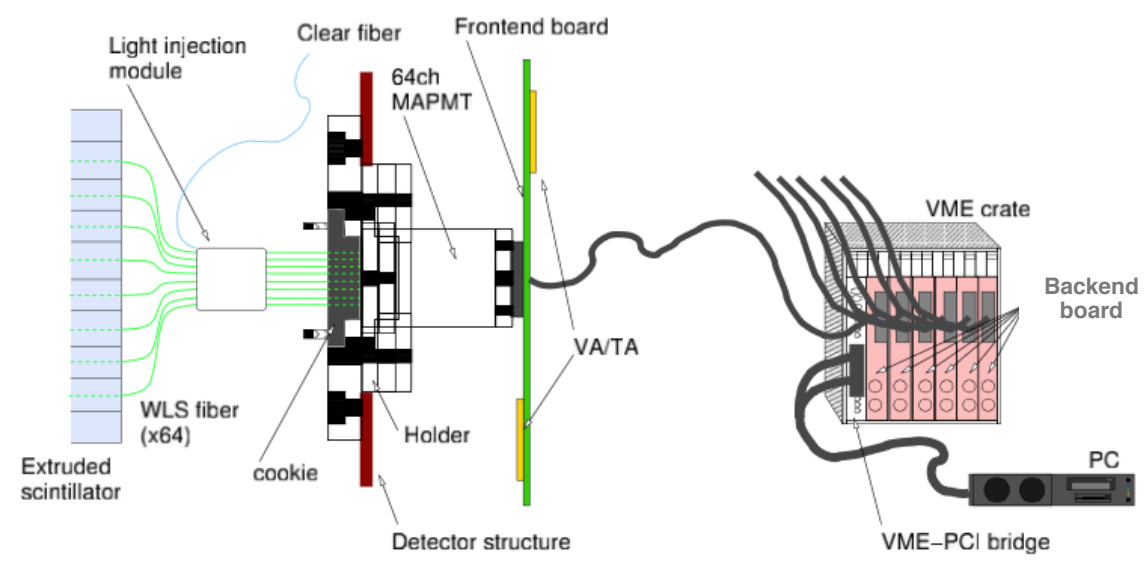

Figure 2: A schematic view of the readout electronics. The WLS fibres are bundled and inserted to the photoelectric surface of the 64ch MAPMT. The FEB to which two VA/TA ASICs are mounted are attached to the MAPMT directly. The FEB is controlled by the BEB inserted to the $9 \mathrm{U}$ VME crate through $4 \mathrm{~m}$ cable. [5]

CMOS circuit including charge sensitive preamplifier, slow shaper and sample\&hold. It corresponds to 32 multi-channel inputs and has a serialized one output line by multiplexer. "TA" is a ASIC with a fast trigger function, which consists of fast shaper and level sensitive discriminator. Each output is summarised to one output via OR circuit on the latter part. TA output signals are sent to a trigger board (TRGB) through a back-end board (BEB) so as to be judged by the Muon or Neutron criteria and the trigger signal is generated. The trigger output signal from the TRGB is distributed to each BEB to inform timing of sample\&hold. Then VA output signals are converted to digital values by a Flash ADC and accumulated to a Field Programmable Gate Array (FPGA). Finally the ADC data are transferred to our DAQ server via VME bus. 


\section{Motivation \& Development}

We have three main reasons to develop a new DAQ system in order to utilize the SciCRT as an effective cosmic-ray telescope.

Firstly there is the problem about dead time to acquire ADC data. It takes $\sim 1.2 \mathrm{msec}$ to acquire one ADC data on the BEB. This is related to the readout processing via VME bus. Therefore the maximum transfer speed of ADC data is limited to $\sim 1 \mathrm{kHz}$. Secondly each TA output line is affected by the readout noise while the BEB deals with the process to obtain the ADC data. According to the problem, it is impossible to record all the triggered events. Moreover the Anti signals from veto counters have $\sim 1 \mathrm{msec}$ dead time. This also makes the sensitivity to neutrons lower. Thirdly the DAQ system should be optimised to our severe experimental environment, 4,600 $\mathrm{m}$ a.l.s, which was not taken into account at the accelerator experiment.

Therefore we developed a brand-new fast readout BEB within the Open-It project [6]. Our new BEB is based on a data transmission technique, SiTCP [7]. SiTCP is a hardware-based network processor which can be implemented on a FPGA. SiTCP can treat Transmission Control Protocol/Internet Protocol (TCP/IP) and Remote Bus Control Protocol (RBCP). In the case of our new $\mathrm{BEB}, \mathrm{TCP} / \mathrm{IP}$ is applied for the data transmission to the DAQ PC and RBCP is used to write a register, e.g. threshold values.

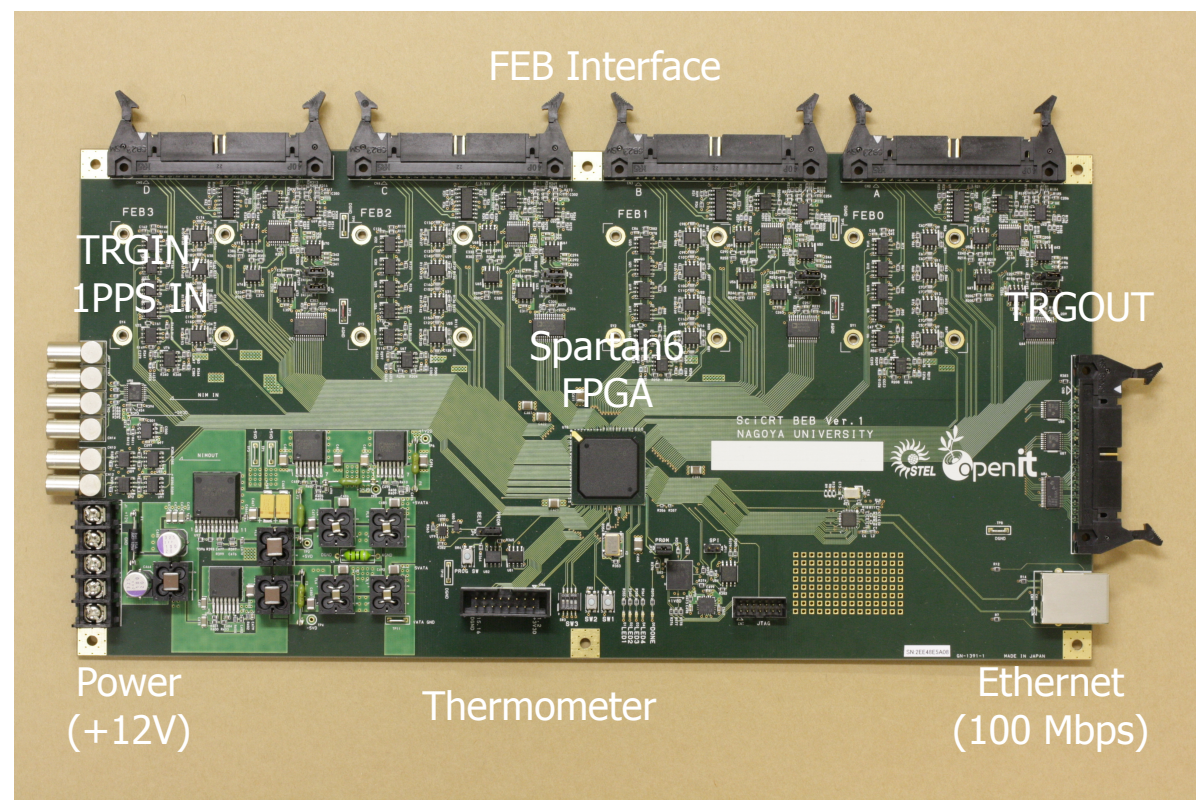

Figure 3: An image of the new SciCRT BEB. The new BEB readouts four FEBs. Total eight TA output signals are sent to a TRGB with LVDS interface. Trigger signals from the TRGB and $1 \mathrm{~Hz}$ common reset signals which are explained in the following text are injected with NIM interface.

\section{Basic Performance Evaluation}

Before the new BEBs are installed to the SciCRT, we performed several basic evaluations in Nagoya. Here we will refer to a few results in the following contexts. 
At first, we evaluated a noise level for the pedestal distribution. The common mode noise can be subtracted by off-line analysis. After the analysis, we got a fact that the noise level for each propagation mode (common mode noise and normal mode noise) become 0.4 and 1.9 ADC counts. Therefore we can conclude that the normal mode noise is dominant for our new BEB, which is equivalent to 0.3 p.e. This definitive noise level is sufficiently low and equal to it for the old BEB developed for the SciBar detector [8].

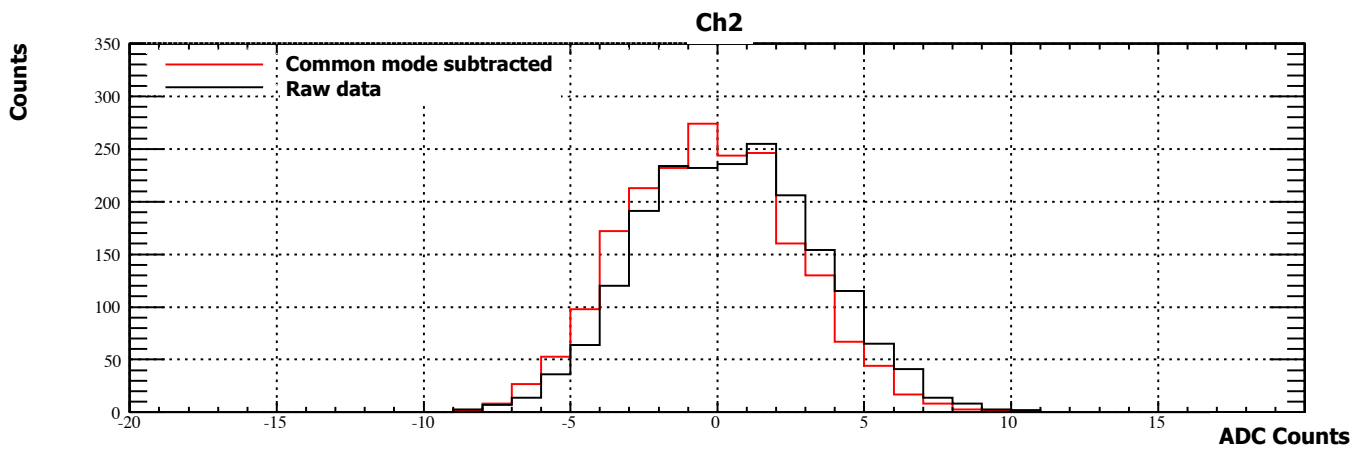

Figure 4: A typical channel of the pedestal distribution with the black solid line is shown compared with the common mode subtracted distribution with the red solid line. The common mode subtracted distribution is almost overlapped with the raw data distribution so that we cannot distinguish these two distributions.

We measured the ADC linearity for the new BEB. The dummy VA output signals which are common with 64 channels are injected to the input line on the new BEB. The input voltage ranged from 0 to $80 \mathrm{mV}$. As a result of the analysis, the linearity is estimated to be within $2 \%$ over the range of our flash-ADC.

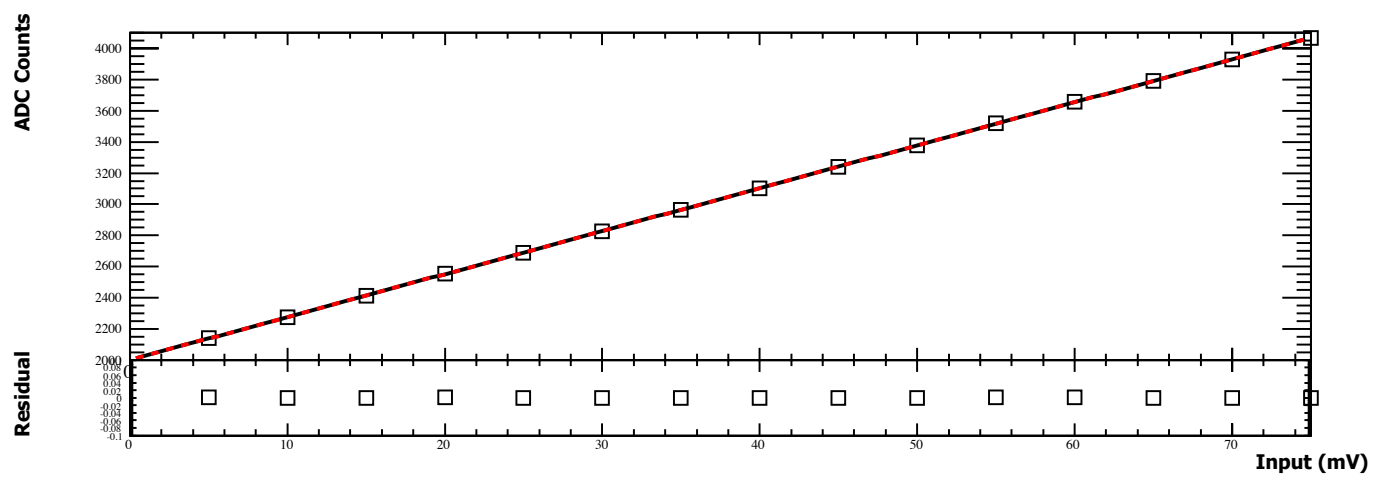

Figure 5: An ADC linearity for different input voltages from 0 to $80 \mathrm{mV}$. The values of the vertical axis show the averaged ADC counts. Each plot includes the error bar. The figure below shows a residual between each plot and a linear fit. These plots are distributed within $2 \%$.

A crosstalk was also measured for our readout electronics including the FEB without the MAPMT. The crosstalk rate for the MAPMT on the photoelectric surface is $\sim 3 \%$. Therefore it is sufficient that our requirement for the readout electronics is less than 3\%. As the result of the measurement, the crosstalk rate for the electronics is estimated to be less than $0.3 \%$ as shown in 
Fig.6. The highest crosstalk rate is $0.3 \%$ at the previous channel $(\mathrm{CH} 2)$ to the injected channel $(\mathrm{CH} 3)$ in the sequence of VA readout.

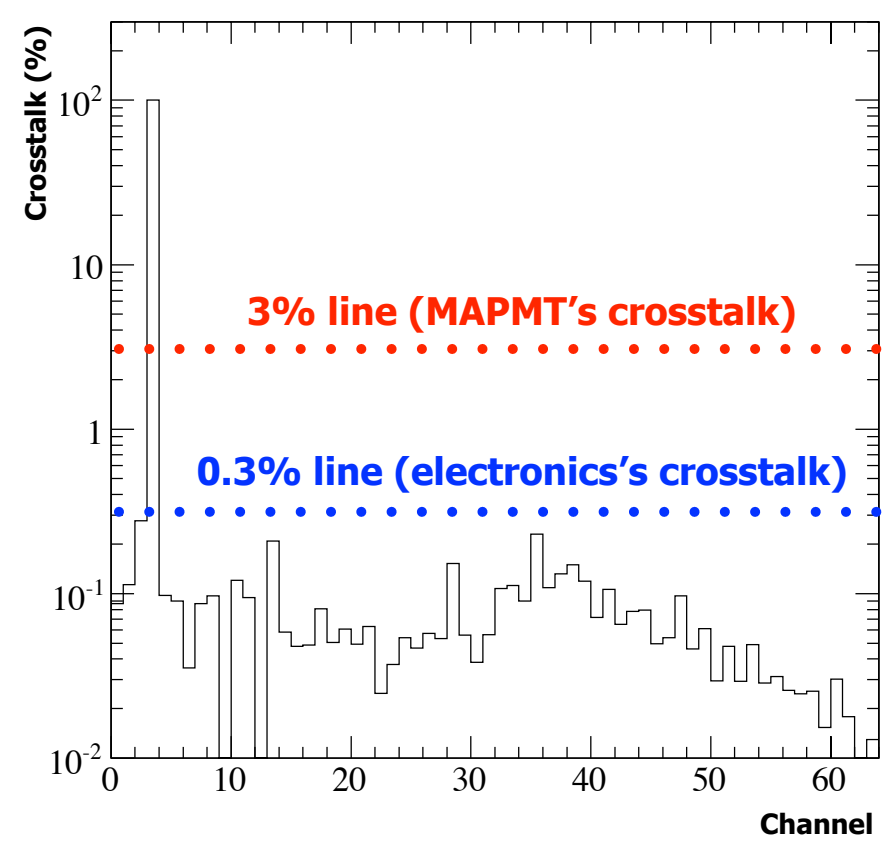

Figure 6: A percentage of the crosstalk for the readout electronics without the MAPMT. The pulse injected channel $(\mathrm{CH} 3)$ is shown as $100 \%$ crosstalk.

\section{Installation \& Configuration}

We developed two new "Ver1.0" BEBs in March, 2014. After we evaluated the basic performance at Nagoya and tested at Mt. Sierra Negra between October to November, we developed eight "Ver2.0" BEBs for the installation to the SciCRT.

We installed new BEBs for Muon and a part of Neutron, SB3 in June, 2015. According to the basic evaluation, these new BEBs are inserted to an assembled rack and installed at the outside of the dark box. We also use the 9U VME-based TRGB for combining the hardware trigger processes by accumulating TA signals. To combine each event obtained by each BEB, a event build counter is used on a BEB. Each BEB counts the event build counter with $1 \mathrm{MHz}$ accuracy independently. A General Purpose Input/Output (GPIO) module sends $1 \mathrm{~Hz}$ common pulse to BEBs to reset the event build counter shown in Fig.7.

According to the faster transmission speed to the DAQ PC, the dead time is improved up to $\sim 110 \mu \mathrm{sec}$. Therefore the readout speed of the new BEB becomes about 10 times higher than the old BEB. The new BEB has also no readout noise on the TA hit lines. Then there is no Anti dead time caused by the electronics. 


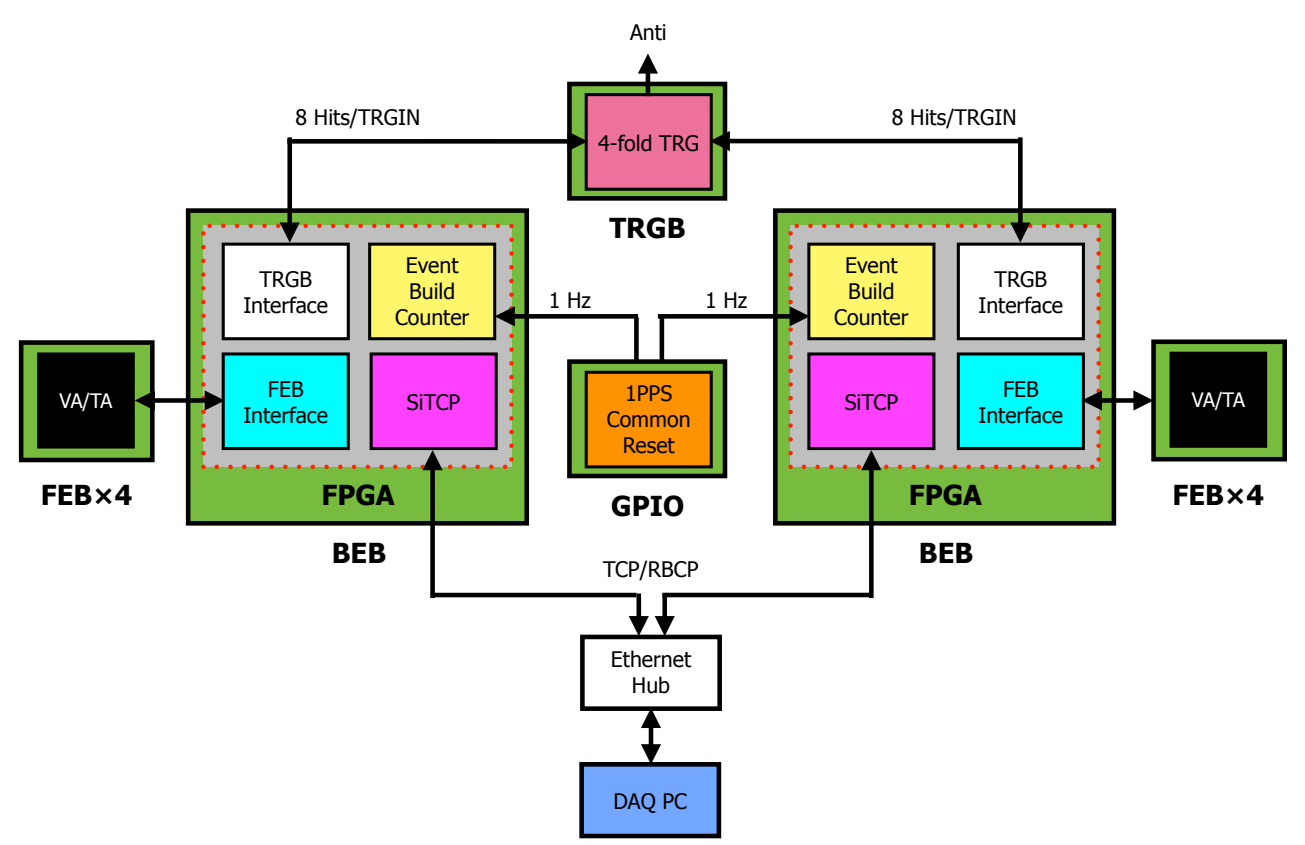

Figure 7: A schematic diagram of the new Muon DAQ system.

\section{Summary}

The SciCRT is a multi-purpose cosmic-ray detector based on the SciBar detector developed for the $\mathrm{K} 2 \mathrm{~K}$ neutrino oscillation experiment. To further improve the performance of the SciCRT, we developed a new fast readout BEB via SiTCP/100 Mbps named "Ver1.0". By obtaining the pedestal data, we found that the normal mode noise becomes $\sim 2$ ADC Counts $(0.3$ p.e. equivalent level). The ADC linearity is ensured within $2 \%$ in the range of our use. The crosstalk rate for our electronics is less than $0.3 \%$. Finally, the readout speed of the ADC data is enhanced to 10 times higher than the old DAQ system. There is also no readout noise when the ADC data is readout. Then we produced eight new "Ver2.0" BEBs in March 2015 and installed the BEBs to the Muon and the Neutron SB3 of the SciCRT from June to July. The new DAQ system has operated the data continuously at present.

\section{Acknowledgment}

We are grateful for the group of the SciBar and SciBooNe experiment to allow us to use the SciBar as a cosmic-ray detector. We are also grateful for the Instituto Nacional de Astrofísica, Óptica y Electrónica (INAOE) to allow us to continue the experiment at the top of Mt. Sierra Negra. This work was supported by Grants-in-Aid for Scientific Research (B) 22340054, Scientific Research (C) 23540348 and Fellows 24340054 from JSPS. Grants from CONACyT (181879-I) and PAPIIT-UNAM (IN107911) are also acknowledged. This work is also supported in a part by the joint research program of the Solar Terrestrial Environment Laboratory (STEL), Nagoya University. We also thank advice and help for the development to Open-It consortium. 


\section{References}

[1] K. Koga et al., Measurement of high-energy neutrons at ISS by SEDA-AP, Astrophys. Space Sci. Trans., 7, 411-416 (2011)

[2] David J. Lawrence et al., Detection and characterization of 0.5-8 MeV neutrons near Mercury: Evidence for a solar origin, JGR, VOL. 119, 5150-5171 (2014)

[3] K. Watanabe, Solar neutron events associated with large solar flares in solar cycle 23, PhD thesis, Department of Physics, Nagoya University (2005)

[4] T. Sako et al., Long-lived solar neutron emission in comparison with electron-produced radiation in the 2005 september 7 solar flare, APJ 651, 69-72 (2006)

[5] S. Yamamoto, Master Thesis, Kyoto University (2003)

[6] http://openit.kek.jp

[7] T. Uchida, Hardware-based tcp processor for gigabit ethernet, IEEE Transactions on Nuclear Science 55, 1631-1637 (2008)

[8] M. Yoshida et al., Development of the Readout System for the K2K SciBar Detector, IEEE Transactions on Nuclear Science, Vol.51, No.6, 3043-3046 (2004) 